\title{
SPARSE MULTICHANNEL SOURCE SEPARATION USING INCOHERENT K-SVD METHOD
}

\author{
Vahid Abolghasemi, Saideh Ferdowsi, and Saeid Sanei
}

\author{
NICE Research Group, Faculty of Engineering and Physical Sciences, University of Surrey, UK \\ Emails: \{v.abolghasemi, s.ferdowsi, s.sanei\}@surrey.ac.uk
}

\begin{abstract}
In this paper the problem of sparse source separation of linear mixtures is addressed. We propose to apply K-SVD, which is a leading dictionary learning method, for this purpose. Further, a modified gradient-based K-SVD scheme for incoherent dictionary learning and source separation is proposed. The promising results on random synthetic signals reveal the ability of this technique for utilizing in source separation framework. We also suggest BOLD detection fMRI as an application for this method. The preliminary results confirm the successful separation of this type of data.
\end{abstract}

Index Terms - Blind source separation, dictionary learning, sparse component analysis, singular value decomposition

\section{INTRODUCTION}

Blind Source Separation (BSS) which means decomposition of a mixture into a mixing matrix and original source signals is considered as a fundamental problem in signal processing community. This kind of matrix factorization has various applications such as in speech processing, communications, and biomedical signal and image processing [1]. A linear mixture model can be generally represented as follows

$$
X=A S+V,
$$

where $A \in \mathbb{R}^{m \times n}$ is an unknown mixing matrix, $S \in \mathbb{R}^{n \times T}$ consists of $n$ sources (rows) each containing $T$ samples. Also, the additive noise denoted by $V$ is a real $m \times T$ matrix.

Depending on the number of mixtures $m$ (also called sensors) and sources $n$, the BSS problem is mainly divided into three categories: exact-determined $(m=n)$, under-determined $(m<n)$, and over-determined $(m>n)$. Most of the existing factorization methods in the literature need to be modified when dimensions of the given mixture vary from one category to another. The factorization in (1) is basically an ill-posed problem and needs additional constraints to become tractable. One of the most popular methods for solving the over-determined and exact-determined BSS is Independent Component Analysis (ICA) [1] which applies statistical independency of the sources as a constraint. In ICA the aim is to find $A$ and $S$ by maximizing the statistical independency of the estimated components. Nonnegative Matrix Factorization (NMF) [2] is also another method which imposes nonnegativity constraint on all elements in the factorization process. Sparsity is a useful constraint which has been considered for BSS problem [3], too. A sparse signal has merely

This work is supported by The Leverhulme Trust. few active (non-zero) samples compared to the total length. In Sparse Component Analysis (SCA) it is assumed that the source matrix $S$ has far less number of non-zeros and one seeks the sparsest $S$ which satisfies (1). It has been recently observed that not always independency or nonnegativity exist for the sources of interest, whereas sparsity may exist. For instance, preliminary results in fMRI application, where ICA has been dominantly used as the separation technique, reveals the advantage of SCA rather than ICA [4]. Another family of sparsity-based factorization techniques are Dictionary Learning (DL) methods. Considering columns of $X$ as a set of training signals (e.g. face images), the aim is to sparsely represent them over a dictionary $D$. DL is mainly considered to be an exact-determined or under-determined factorization. There are different DL methods such as MAP [5], MOD [6], and K-SVD [7]. The latter is a leading method and is employed in this paper.

Here, we present the similarities between DL and BSS and propose to use the K-SVD algorithm for BSS. We also propose an incoherent K-SVD scheme to decrease the coherence in the columns of dictionary. The results are encouraging and verify applicability of the proposed idea. In addition, it is seen that K-SVD can be used successfully for all three categories of BSS problem without any modification, subject to having sparse sources.

In the next section, we first introduce the K-SVD algorithm. Then, the proposed incoherent K-SVD is described. Finally, applicability of K-SVD and modified K-SVD to BSS problems is argued. In section 3, the experimental results are presented. In section 4 we conclude the paper.

\section{PROBLEM FORMULATION}

\subsection{K-SVD}

K-SVD which was firstly introduced by Aharon [7] has become a popular DL method for deriving dictionaries which can sparsely represent the given signal. It is a generalization of K-means clustering method and attempts to design a dictionary $D \in \mathbb{R}^{m \times n}$ from $N$ number of training signals of length $m$. This problem can be mathematically expressed as the following alternating minimization:

$$
\min _{D, S}\|X-D S\|_{F} \quad \text { s.t. } \quad\left\|s_{: i}\right\|_{0} \leq T_{0}, i=1,2, \ldots, N
$$

where $\|\cdot\|_{F}$ is the Frobenius norm and is a measure of the error between $X$ and $D S$. The term $\|.\|_{0}$ is $\ell_{0}$-norm which represents the support of its argument (number of active components). $s_{: i}$ refers to the $i$ th column of matrix $S$. The K-SVD algorithm is composed of two major steps; sparse coding and 
dictionary update. Sparse coding is simply applying any existing pursuit algorithm, such as Orthogonal Matching Pursuit (OMP), to all columns of $X$ to find sparse vectors $s_{: i}$, when the dictionary $D$ is fixed. Alternatingly, (2) is solved to derive $D$, when $S$ is considered fixed. In K-SVD the innovation is in the second part, where an SVD-based method is proposed to update both dictionary elements and non-zero coefficients of $S$ in a column-wise fashion. Consider the $k$ th column of the dictionary denoted by $d_{: k}$, and the corresponding coefficients in $S$ ( $k$ th row of $S$ ), denoted as $s_{k:}$. The Frobenius norm in (2) can then be expanded as follows [7]:

$$
\begin{aligned}
\|X-D S\|_{F}^{2} & =\left\|X-\sum_{j=1}^{n} d_{: j} s_{j:}\right\|_{F}^{2} \\
& =\left\|\left(X-\sum_{j \neq k} d_{: j} s_{j:}\right)-d_{: k} s_{k:}\right\|_{F}^{2} \\
& =\left\|E_{k}-d_{: k} s_{k:}\right\|_{F}^{2} .
\end{aligned}
$$

The idea in K-SVD is to apply singular value decomposition (SVD) to $E_{k}$ in order to update $d_{: k}$ and $s_{k}$ : to minimize the norm. However, this step cannot be applied as straightforward as what just mentioned and needs minor modification. We should consider the sparsity constraint when updating $s_{k}$ : In order to do this, a simple procedure is proposed in [7]. We define $\omega_{k}$ as the group of indices where $s_{k i}$ is non-zero. This refers to $\left\{x_{: i}\right\}$ that only uses the atom $d_{: k}$ :

$$
\omega_{k}=\left\{i \mid 1 \leq i \leq N, s_{k i} \neq 0\right\} .
$$

The squeezed row vector $\tilde{s}_{k}$ : can then be obtained by multiplying $s_{k}$ : by a matrix $\Omega_{k}$ of size $N \times\left|\omega_{k}\right|$, which has ones on the $\left(\omega_{k}(i), i\right)$ th entries and zeros elsewhere: $\tilde{s}_{k:}=s_{k} \Omega_{k}$. Similarly, $E_{k}$ and $X$ are converted to $\tilde{E}_{k}=E_{k} \Omega_{k}$ and $\tilde{X}_{k}=X \Omega_{k}$, respectively. Now, we can amend the minimization of (3) to

$$
\left\|E_{k} \Omega_{k}-d_{: k} s_{k} \Omega_{k}\right\|_{F}^{2}=\left\|\tilde{E}_{k}-d_{: k} \tilde{s}_{k:}\right\|_{F}^{2}
$$

which is minimized by applying SVD to $\tilde{E}_{k}$, that gives us $\tilde{E}_{k}=$ $U \Lambda V^{T}$. Then, the $k$ th column of $D$ is updated as $\hat{d}_{k}=u_{: 1}$, where $u_{: 1}$ is the first column of $U$. The row vector $\tilde{s}_{k}$ : is also updated by multiplying the first column of $V$ by $\lambda_{11}$. Note also that a normalization step on all columns of $D$ is required in each iteration.

\subsection{Proposed Gradient-based Optimization for Atom Update}

Incoherency of the atoms (columns) in the dictionary is a desired feature for almost all dictionary learning methods. That ensures the leaned atoms to be as distant as possible and prevents the atoms to be similar. Here, we suggest to apply our previously proposed method in [8] to optimize the obtained dictionary in the standard K-SVD algorithm, at each iteration. The proposed method can be briefly described as follows.

Let us assume that $D_{(i)}$ is the dervied dictionary at $i$ th iteration of standard K-SVD. In order to optimize $D_{(i)}$ to decrease the coherence in columns, the following cost function is proposed ${ }^{1}$ :

$$
D_{(i)}=\arg \min _{D_{(i)}}\left\|D_{(i)}^{T} D_{(i)}-I\right\|_{F}^{2}
$$

where $I$ is the identity matrix of size $n \times n$. In order to minimize the above problem, a gradient-descent strategy is proposed, which iteratively minimizes (6), while updating $D_{(i)}$. Taking the gradient of $E:=\left\|D_{(i)}^{T} D_{(i)}-I\right\|_{F}^{2}$ and inserting it into $D \leftarrow D-\eta \nabla E$, results in:

$$
D_{(i)}^{(\text {new })}=D_{(i)}^{(\text {old })}-\eta D_{(i)}^{(\text {old })}\left(D_{(i)}^{T(\text { old })} D_{(i)}^{(\text {old })}-I\right) .
$$

Here, $\eta>0$ is the step-size which controls the convergence behavior of the algorithm. After updating all dictionary columns in the standard K-SVD, the above update rule is applied and repeated for a few times to optimize the dictionary. The columns of $D$ are normalized after implementing (7), too.

\subsection{Application to Blind Source Separation}

A careful consideration of the aforementioned BSS problem in section 1 and the K-SVD in 2.1 and 2.2 reveals some similarities which one may think of it as the applicability of $\mathrm{K}-\mathrm{SVD}$ to solve the BSS problems (of course subject to some constraints, e.g. sparsity). This section is devoted to highlighting these connections and shows the possibility of applying the proposed and standard K-SVD methods to BSS problems.

Assume we have $n$ sources of length $N$ with the same sparsity bound. This means if we arrange the sources in a row-wise manner into $S$, each column would have at most $T_{0}$ non-zeros. The sources we describe here are exactly similar to what we achieve in DL when solving (2). Assume further, we only have the knowledge about $m$ mixtures $X \in \mathbb{R}^{n \times N}$ which are viewed as $N$ training signals in DL. In fact, each column of $X$ is considered as one training signal in DL, while each row of $X$ is considered as a mixture in BSS. Although these are two different interpretations of $X$, they do not change the problem formulation. Finally, dictionary $D$ in DL has the same intuition as the mixing matrix $A$ in BSS problem. If no constraint has to be imposed on $A$, the standard K-SVD is applicable for a sparse BSS problem. However, for the case of mixing matrices with small coherence, the proposed modified scheme is suitable. Finally, to keep the consistency in notation the number of training signals, $N$ (in DL), is replaced with $T$, which is the number of samples of each source.

We note here that applying K-SVD to the given mixtures $X$ may cause row permutation of $S$ and column scaling of $A$, like any other separation technique. However, since we deal with column normalized mixing matrices this problem is alleviated to sign permutation only. In addition, the results of our simulation experiments show that the proposed application of K-SVD works well for all three cases of exact-determined, over-determined, and under-determined BSS.

\section{EXPERIMENTS}

We divide this section into two parts: synthetic and real data experiments. In addition, we compare the results of the

\footnotetext{
${ }^{1}$ See [8] for an extended discussion on this method.
} 
proposed method and those of obtained from HALS-SCA technique [2] which is used for separation of sparse underdetermined mixtures. A comparison between the proposed method, ICA method [1] and NMF technique [9] is also demonstrated for the case of real over-determined fMRI sequences including the Blood Oxygenated Level Dependent (BOLD).

\subsection{Synthetic Data}

In the first experiment we randomly generated a sparse source matrix $X$ of size $10 \times 1000$. Then, a random overcomplete mixing matrix of size $8 \times 12$ was created with all columns normalized to one. We also added a Gaussian noise of zero mean and 0.01 variance to the mixing model. Then, the proposed modified K-SVD and standard K-SVD algorithms with 500 iterations and OMP sparse coder was applied to $X$ to estimate the mixing matrix $\hat{A}$ and sources $\hat{S}$. The step-size value $\eta=0.01$ was selected in the optimization step. Considering sign permutations between columns of $A$ and $\hat{A}$, the Signal to Interference Ratio (SIR) defined as $S I R_{d B}=20 \log \frac{\|A\|}{\|A-\hat{A}\|}$ was recorded. We repeated these simulations for 10000 random ensembles of $A$ and $S$ with different sparsity levels from 1 to 6 . The average SIR against the sparsity level is given in Figure 1. Similar curves as a result of applying HALS-SCA [2] and Method of Optimal Directions (MOD) [6] were also plotted on the same graph. It is seen that both K-SVD and modified K-SVD can well estimate the mixing matrices and sources, and outperform other methods. Moreover, when the number of non-zeros increases while other dimensions are fixed the reconstruction performance is degraded, as expected.

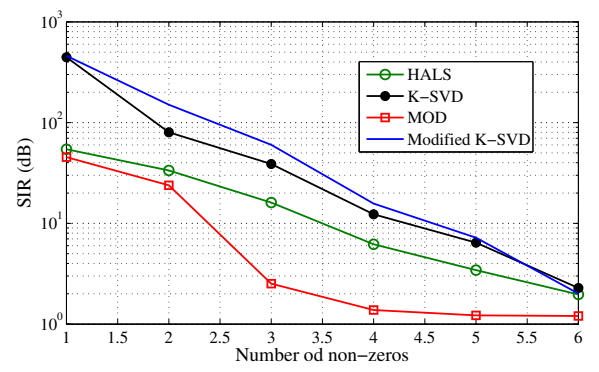

Fig. 1. Average SIR for mixing matrix against number of non-zeros.

Next, in order to show the efficiency of K-SVD for overdetermined case, we designed an experiment with a random $A$ of size $15 \times 10$ and a source matrix $S$ of size $10 \times 500$. We chose a severe sparse condition on $S$, where only $60 \%$ of each column's coefficients were zero. We also added Gaussian noise of zero mean and 0.05 variance. The K-SVD algorithm was then applied 10000 times to such ensembles. The average SNRs obtained for $A$ and $S$ are $72.62 \mathrm{~dB}$ and 58.36 $\mathrm{dB}$, respectively. Figure 2 (a) represents one of the original sources (first row of $S$ ) and the corresponding recovered source. The accuracy of reconstruction is clearly observed. In addition, we have shown in Figure 2 (b) and (c) the numerical values of the first two columns of the original mixing matrix and their corresponding columns in the estimated matrix. Again, the high accuracy in recovery is observed. Furthermore, we observed that when the level of sparsity decreases, a larger number of iterations is needed to correctly recover the

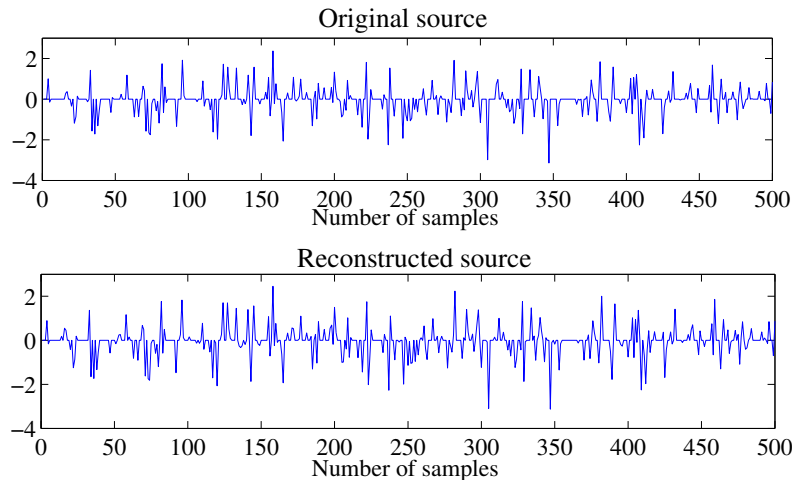

(a)

$\left(\begin{array}{cc}-0.1576 & 0.3795 \\ -0.2340 & -0.2905 \\ 0.4817 & -0.1341 \\ -0.0651 & 0.0821 \\ 0.0093 & -0.3532 \\ -0.2105 & -0.4894 \\ -0.0801 & 0.2506 \\ -0.4821 & 0.2502 \\ -0.0098 & -0.1156 \\ -0.2170 & 0.1615 \\ -0.2252 & 0.2189 \\ 0.5388 & -0.3140 \\ -0.0834 & 0.1777 \\ -0.0338 & 0.1503 \\ -0.0678 & -0.1308\end{array}\right)$

(b)

$$
\left(\begin{array}{cc}
-0.1630 & 0.3780 \\
-0.2347 & -0.2894 \\
0.4827 & -0.1282 \\
-0.0687 & 0.0887 \\
0.0108 & -0.3658 \\
-0.2173 & -0.4887 \\
-0.0876 & 0.2566 \\
-0.4797 & 0.2540 \\
-0.0139 & -0.1107 \\
-0.2161 & 0.1674 \\
-0.2245 & 0.2134 \\
0.5347 & -0.2965 \\
-0.0884 & 0.1743 \\
-0.0193 & 0.1496 \\
-0.0645 & -0.1395
\end{array}\right)
$$

(c)
Fig. 2. Results of an over-determined case experiment using KSVD. (a) Original and recovered source ensemble, and (b) original and (c) recovered mixing matrix components.

sources. One appropriate way to terminate the K-SVD algorithm is to set up the stopping criterion based on the difference of the error between two successive iterations.

In another attempt, we conducted an extensive simulation to observe the separation quality of the proposed method for different dimensions. 100 trials were generated at each point on a plane of $(m, n)$, for $T=512$, where $n \in[5125]$, and $m \in[535]$. This experiment was implemented for the sparsity level of $q=3$. Non-zeros of the sparse signals in each trial were drawn from random Gaussian distribution. The result is demonstrated in Figure 3. Darker and brighter regions represent higher and lower percentage of success, respectively. A successful recovery was reported when $\hat{A}$ and $\hat{S}$ were close to true $A$ and $S$, with a small tolerance. It is also found from Figure 3 that increasing the number of columns (increasing the degree of under-determinacy) reduces the success rate,

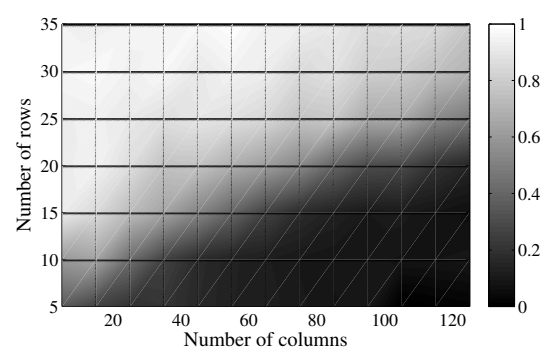

Fig. 3. Success rate plane for $q=3$. 
the algorithm performance is acceptable for moderate underdetermined cases, though.

\subsection{Real Data}

\subsection{1. fMRI data set}

In order to inspect the results of applying the proposed method to real scenarios we chose fMRI application. An auditory data set was taken from the SPM website [10], which consists of the brain images acquired by a $2 \mathrm{~T}$ scanner with 96 scans in total. Each scan consists of 64 successive slices $\left(64 \times 64 \times 64,3 \times 3 \mathrm{~mm} \times 3 \mathrm{~mm}^{3}\right.$ voxels $)$ with the scan to scan repeat time (TR) of 7s. 96 data acquisition were processed on a single subject, in blocks of 6 , giving 16 42s blocks. The condition for successive blocks alternated between rest and auditory stimulation starting with rest. The auditory stimulation is by using bi-syllabic words presented binaurally at a rate of 60 per minute. The aim is to detect the BOLD area in the brain using the proposed separation technique.

\subsubsection{Results}

After several pre-processing steps the fMRI mixture $X$ is formed as a matrix of size $96 \times 75075$, where each row represents the spatial information and each column shows the temporal variations of the corresponding voxels. We applied K-SVD on this matrix and chose the number of sources to be 50. 1000 iterations were used and the level of sparsity in each column was set to 5. We also applied Infomax ICA and NMF method proposed in [9] to the same fMRI data to analyze and compare the results. Figure 4 demonstrates the results of these experiments. Comparing Figure 4 (a), (b) and (c) reveals that although ICA performs better in detecting the timecourse, correct BOLD detection using K-SVD is an encouraging outcome. The sparse BOLD is visible from Figure 4 (a) and also (b) which is an advantage of the proposed method [4]. However, the presented results here are preliminary and further work is required for measuring the quality of BOLD detection using K-SVD.

\section{CONCLUSION}

In this paper K-SVD has been used for separation of sparse sources from a given mixture. A modified incoherent K-SVD algorithm was proposed which attempts to produce dictionaries with less coherent atoms. Although K-SVD is a dictionary learning method, we empirically proved that it is also a practical tool for the sparse source separation problems. We stated the similarities between DL and sparse source separation to support the proposed idea. The simulation results, though, evidenced the strengths and advantages of K-SVD for all three cases of over-determined, exact-determined, and under-determined mixtures. Further quantitative and statistic analysis are required to accurately assess the strength of the proposed method on fMRI data, and/or other similar applications.

\section{REFERENCES}

[1] A. Hyvärinen, J. Karhunen, and E. Oja, Independent Component Analysis, Wiley-Interscience, May 2001.

[2] A. Cichocki, R. Zdunek, A. H. Phan, and S. Amari, Nonnegative Matrix and Tensor Factorizations: Applications to Exploratory Multi-way Data Analysis and Blind Source Separation, John Wiley, 2009.
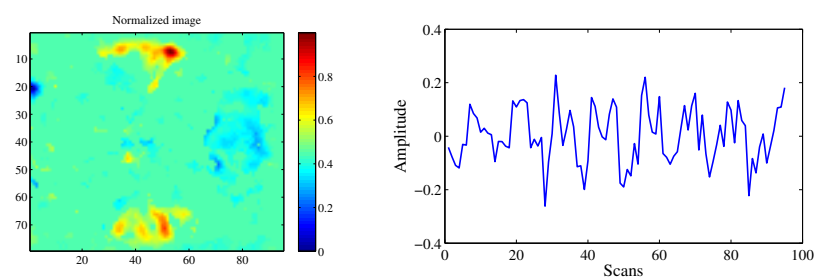

(a) K-SVD
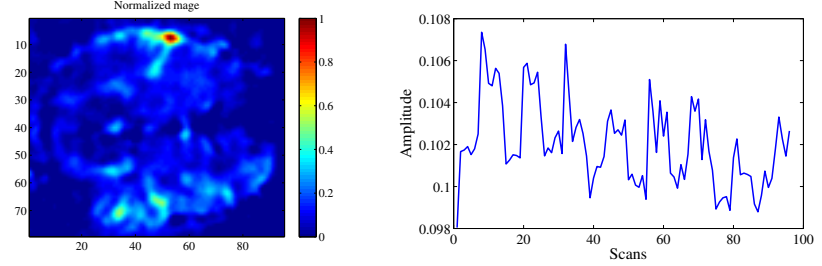

(b) NMF
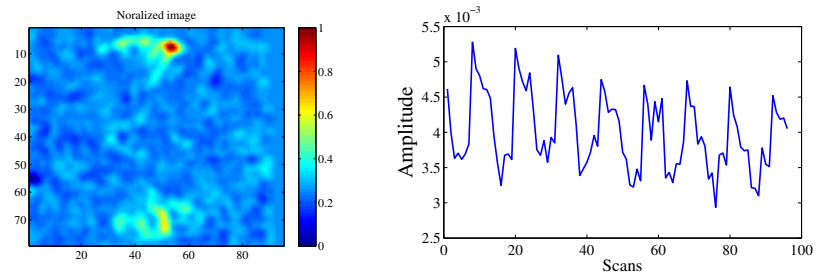

(c) ICA

Fig. 4. Results for the auditory fMRI data separation.

[3] M. Zibulevsky and B. A. Pearlmutter, "Blind source separation by sparse decomposition," Neural Computation, vol. 13, pp. 863-882, 1999.

[4] I. Daubechies, E. Roussos, S. Takerkart, M. Benharrosh, C. Golden, K. D'Ardenne, W. Richter, J. D. Cohen, and J. Haxby, "Independent component analysis for brain fMRI does not select for independence," Proceedings of the National Academy of Sciences, vol. 106, no. 26, pp. 10415-10422, June 2009.

[5] B. A. Olshausen and D. J. Field, "Sparse coding with an overcomplete basis set: a strategy employed by v1?," Vision research, vol. 37, no. 23, pp. 3311-3325, December 1997.

[6] K. Engan, S. O. Aase, and J. Hakon Husoy, "Method of optimal directions for frame design," in IEEE International Conference on Acoustics, Speech, and Signal Processing, ICASSP, Washington, DC, USA, pp. 2443-2446, 1999.

[7] M. Aharon, M. Elad, and A. Bruckstein, "K-SVD: An algorithm for designing overcomplete dictionaries for sparse representation," IEEE Trans. on Signal Processing, vol. 54, no. 11, pp. 4311-4322, 2006.

[8] V. Abolghasemi, S. Ferdowsi, and S. Sanei, "On optimization of the measurement matrix for compressive sensing," in 18th European Signal Processing Conference (EUSIPCO2010), Aalborg, Denmark, Aug. 2010, pp. 427-431.

[9] S. Ferdowsi, V. Abolghasemi, and S. Sanei, "A constrained NMF algorithm for BOLD detection in fMRI," in IEEE International Workshop on Machine Learning for Signal Processing (MLSP), Sept. 2010, pp. $77-82$.

[10] Statitstical Parameter Mapping (SPM) homepage, "http://www.fil.ion.ucl.ac.uk/spm/," . 